\title{
Vibration suppression for high speed railway bridges using fluid viscous dampers
}

\author{
M.Tahiri ${ }^{1, *}$ A. Khamlichi ${ }^{2, * *}$, M. Bezzazi ${ }^{1, * * *}$ \\ 1. Department of Physics, Mechanical and Civil Engineering laboratory, Faculty of Sciences and Technology, University \\ Abdelmalek Essaadi, Tangier, Morocco.*tamamed7@gmail.com;***bezzazi@hotmail.com \\ 2. Department STIC, Communication Systems and Detection Laboratory, National School of Applied Sciences, University \\ Abdelmalek Essaadi, Tetouan, Morocco. **khamlichi7@yahoo.es
}

\begin{abstract}
The results of experimental and theoritical investigations of railway bridges have shown the significant dynamic responses exceeding that anticipated on certain railway bridges, such as a resonance phenomenon who have a great effect in the bridge. Alternatively, the use of structural control systems devices might be a very promising solution to attenuate undesirable vibration. The aim of this study, first, is to investigate the posibility of reducing the acceleration in an acceptable level by using fluid viscous dampers. The bridge-damper system is described by a single-degree-of-freedom model. The ,dampers are connected between the bottom surface of the bridge deck and the abutment, Finallay a linearisation model and a comparative study in order to investigate the effect of the nonlinearite of the device in the dynamic response of the system.
\end{abstract}

Keywords: railway bridges, resonance, passive control, fluid viscous dampers, nonlinear damping

\section{Introduction}

The dynamic performance of railway bridges is an increasingly relevant issue in public transport systems due to the construction of new high-speed railway lines and the use of old lines for higher operating train velocities. Fast trains can induce resonant situation in the bridges, as the train velocity approaches the resonant velocity an inadmissible vertical accelerations may occur in the bridge. This can cause passenger discomfort, ballast deconsolidation, a reduction of the service life of the bridge and subsequent risk of derailment. However, it is essential to control the resonant vibration of such structures under the circulation of trains.

Damping devices for structural control can be divided into three mains groups: passive, active and semi-active control systems, in this work the possibility of mitigating the resonant vibration of bridges by introducing passive energy dissipation devices, and in particular fluid viscous damper is investigated. FVDs have been selected because they dissipate energy at a wide scope of frequency ranges, and not only at narrow ranges like TMDs.

A few studies dated in this area, the first documented by Museros and Martinez-Rodrigo[1-3] are concerned with the reducing vertical accelerations in resonant case by using double beam systems. Luu et al [4] treated the same configuration proposed by Martinez by using the $H_{\infty}$ norm in order to determining the optimal damper constant. Recently, the nonlinearity has a great importance in field research; FVDs exhibiting a nonlinear force-velocity relation. Symans [5] suggested a formula to linearize the above nonlinear FVD model for predicting in seismic protection, Martinez et al [6] presented a model in order to find the optimum retrofitting option with nonlinear fluid VS dampers for seismic design.

The aim of this work is to (1) propose a concept for vibration mitigation strategy for railway bridges based on the FVDs and (2) compare the effectiveness of linear and nonlinear fluid viscous dampers in the dynamic response of the retrofitted system.

\section{Present model}

The present model as shown in the figure 1, consist to retrofitting the beam by an inclined Fluid dampers, the dampers are connected between the surface of the bridge deck and the abutements, this creates an eccentricity between the connection point and the of the dampers and the neutral axis of the bridge,

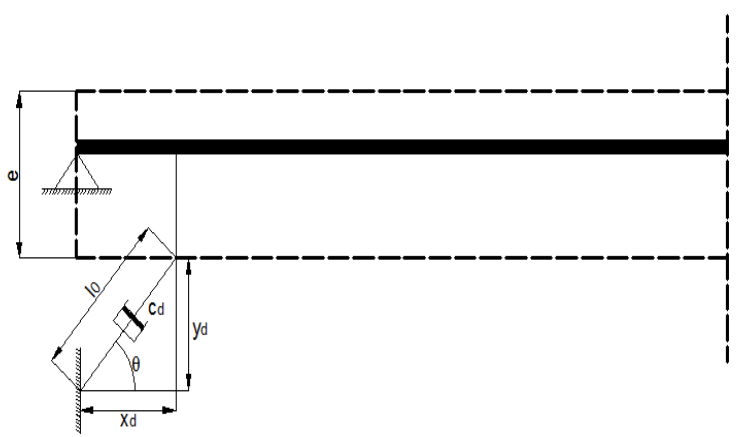

Figure 1 : Single-degree of freedom model of a beam with Fluid Viscous Dampers [6] 
Generally this devices contains a single degree of freeom model, thus the equation of motion of the system expressed as

$$
m \ddot{y}+c \dot{y}+k y=f
$$

Where $y$ is the vertical displacement of the beam $\mathrm{M}$ the mass per unit length of the beam

$\mathrm{C}$ the damping coefficient who contained the damping ratio generated by the fluid viscous damper.

the aim of this work is to investigate the performance of an inclined fluid viscous dampers in order to reduce the dynamic response of the system, and to investigate the effect of nonlinearity by investigating the model proposed by Symans [5] for lineariarisation of the damping force.

Assuming that the dampers do not change the mode shapes of the bridge and in the same case it should be noted that we take only the first mode of vibration.

\subsection{Fluid viscous dampers linearization}

The effectiveness of the use fluid viscous dampers due to their comportment into energy dissipation in wide range frequency, thus the force damping for the case of nonlinear FVD expressed as

$$
F_{d}=c_{\alpha}|\dot{y}|^{\alpha} \operatorname{sgn}(\dot{y})
$$

For the linearization

$$
F_{d}=c_{\text {equ }} \dot{y}
$$

Where $c_{\text {equ }}$ the equivalent damping coefficient.

The principle of this linearization is based in the case of the beam subjected to a cyclic load thus $y=Y \sin (\omega t)$ and by decomposing the nonlinear damping force into Fourier series. Finally, the equivalent damping coefficient expressed as

$$
c_{e q}=\frac{2}{\pi} c_{\alpha} Y^{(\alpha-1)} \omega_{b}^{(\alpha-1)} \frac{\Gamma\left(1+\frac{\alpha}{2}\right) \Gamma\left(\frac{1}{2}\right)}{\Gamma\left(\frac{3}{2}+\frac{\alpha}{2}\right)}
$$

Where $\omega_{b}$ the proper frequency of the beam

$$
\Gamma \text { (.) Gamma function ; } 0.2 \prec \alpha \prec 2
$$

$Y$ The amplitude of the beam at the position of the FVD, furthermore, by decomposing the force due to the moving HSLMA-8 into Fourier series the amplitude can be expressed as

$$
\begin{gathered}
Y(\omega)=C_{1} h_{1} \varphi_{d}\left(\theta_{i}, x_{d, i}\right) \\
\left|C_{1}\right|=\frac{F l}{\pi m\left(x_{N}+l\right)} \frac{2 \cos \left(\frac{\pi l}{x_{N}+l}\right)}{1-\left(\frac{\omega l}{\pi v}\right)^{2}} \sqrt{\left(\sum_{k=1}^{N_{v}} \cos \left(\omega t_{k}\right)\right)^{2}+\left(\sum_{k=1}^{N_{v}} \sin \left(\omega t_{k}\right)\right)^{2}} \\
h_{1}=\frac{1}{\sqrt{\left(\omega_{b}^{2}-\omega^{2}\right)^{2}+4\left(\xi \omega \omega_{b}\right)^{2}}} \\
\varphi_{d}\left(\theta_{i}, x_{d, i}\right)=\frac{\pi e}{L} \cos \left(\theta_{i}\right) \cos \left(\frac{\pi x_{d, i}}{L}\right)+\sin (\theta) \sin \left(\frac{\pi x_{d, i}}{L}\right)
\end{gathered}
$$

$i=1, \ldots N ; \mathrm{N}$ the number of FVDs

Finally the damping ratio in the system retrofitted by fluids viscous damper scan be expressed as

$$
\xi_{d}=\frac{2 \omega_{b}^{\alpha-2} \bar{\Gamma}(\alpha)}{\pi m_{b} l}\left(\left|C_{1}\right| h_{1}\right)^{(\alpha-1)} \sum_{i=1}^{N} c_{d i} \varphi_{d}\left(\theta_{i}, x_{d i}\right)^{(\alpha+1)}
$$

And the total damping ratio can be expressed as

$$
\xi=\xi_{b}+\xi_{d}
$$

\section{Numerical results}

In the following study the properties of the beam is the same treated in the reference [4] and the moving train modeled by the moving load model HSLMA- 8 where properties defined in the Eurocode .

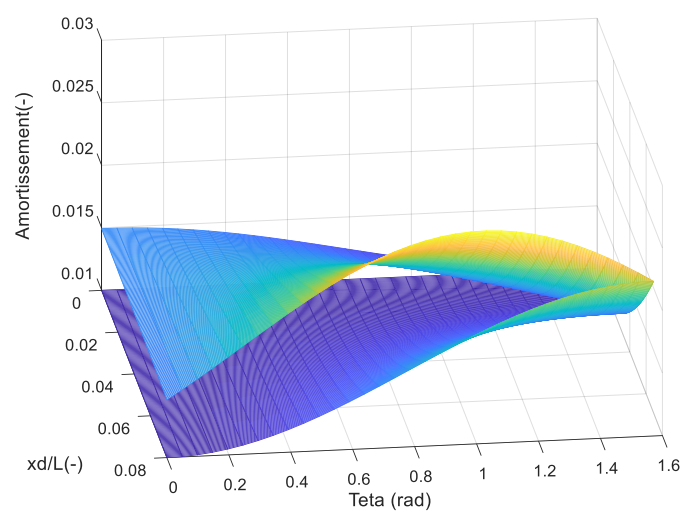

Figure 2: damping ratio as function of the inclination and the position of the $c_{d}=3 M N s / m^{-----} \frac{e}{L}=0,---\frac{e}{L}=0.05$

The total damping equal to $\xi=4.35 \%$ in the linear case, who has a great effect in reducing the acceleration level by $59 \%$ and by $49.6 \%$ for the displacement as shown in the figure 3 , and it should be noted that this percentage of reduction are higher than those founded in the ref [4] by installing a double beam with an FVDs. Thus can be explained the effectiveness of the present model.

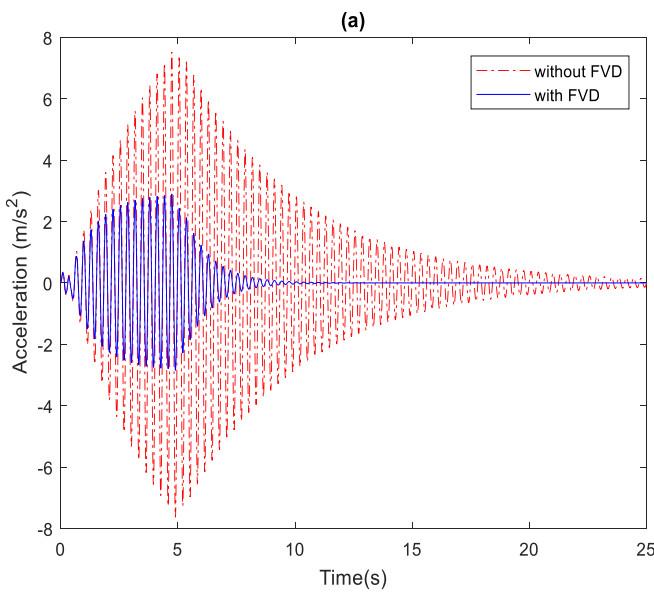

Figure3 (a): acceleration of the beam with and without FVDs 
(b)

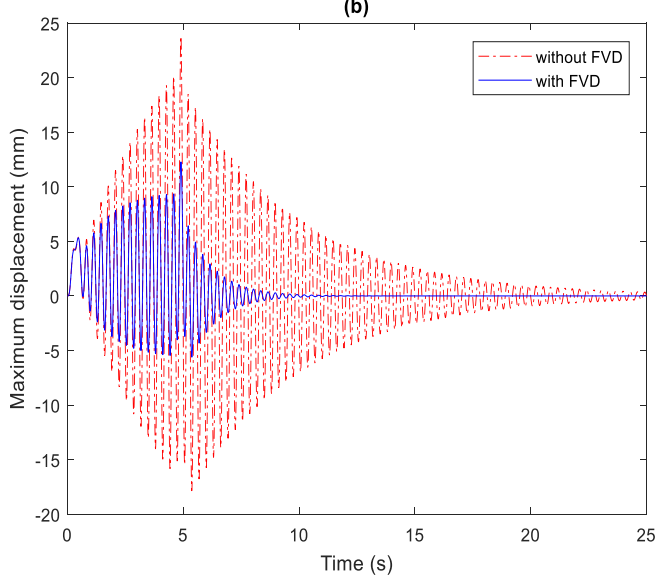

Figure3 (4): Displacement of the beam with and without FVDs

- Effect of nonlinearity :

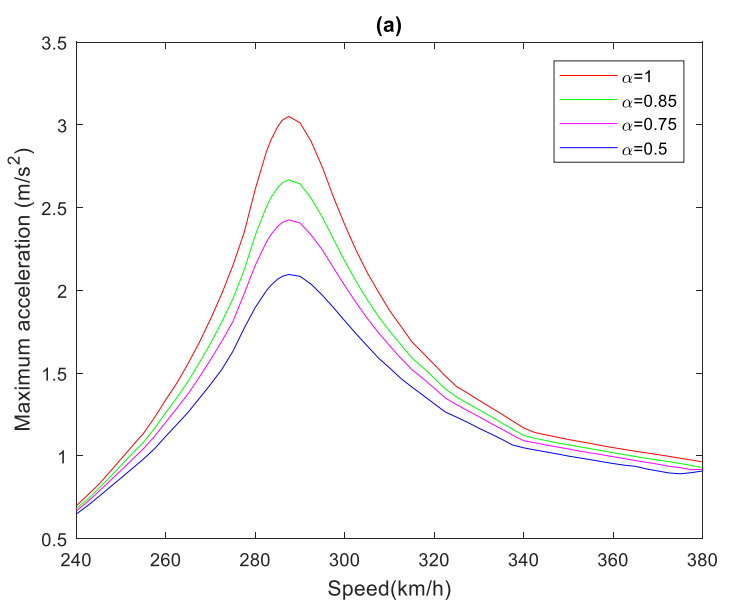

Figure 4(a): acceleration as function of the speed for different $\alpha$

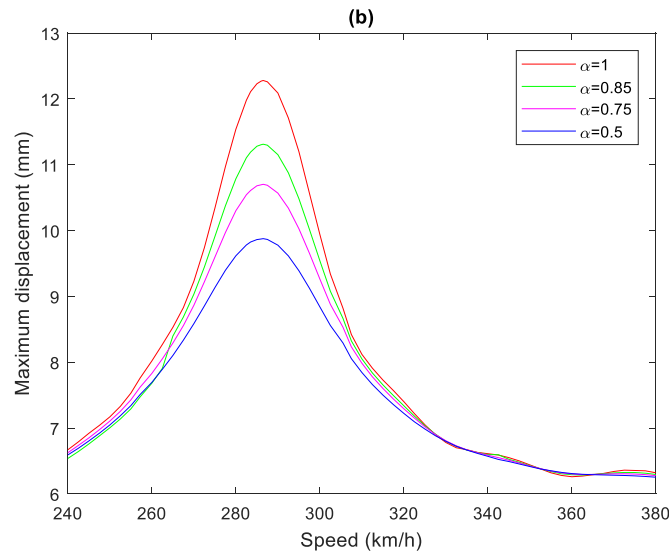

Figure 4(b): Displacement as function of the speed for different $\alpha$

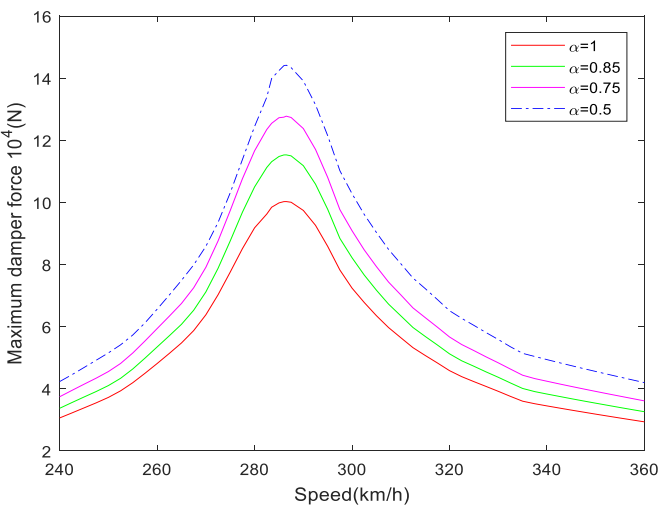

Figure 4(b): Damping force as function of the speed for different $\alpha$

As shown in the figure 4(a)-4(b) as the coefficient $\alpha$ decreases as the dynamic response decreases this same conclusion founded by Symans [5] in the case of retrofitting method for seismic response. In the same case from the figure 5, as the coefficient $\alpha$ decrease the damping force increase from $\alpha=0.5$ we have $30.45 \%$ of reduction between the damping forces in the linear case.

\section{Conclusion}

As demonstrated in the following study the proposed single degree of freedom model has a good performance in the reduction of the dynamic response of the beam in the case of linear and nonlinear Fluid Viscous dampers.

\section{Références}

[1] Lavado, J., Doménech, A., Martínez-Rodrigo, M. D., Dynamic performance of existing high-speed railway bridges under resonant conditions following a retrofit with fluid viscous dampers supported on clamped auxiliary beams. Engineering Structures. 2014 Vol.59, $355-374$

[2] M.D. Martinez-Rodrigo, J. Lavado, P. Museros, Dynamic performance of existing high-speed railway bridges under resonant conditions retrofitted with fluid viscous dampers, Engineering Structures 32. 2010.pp 808-828.

[3] P. Museros, M.D. Martinez-Rodrigo, Vibration control of simply supported beams under moving loads using fluid viscous dampers, Journal of Sound and Vibration 300. 2007 pp, 292-315.

[4] Luu, M., Martínez-Rodrigo, M. D., Zabel, V., Könke, C.,Hळ optimization of fluid viscous dampers for reducing vibrations of high-speed railway bridges. Journal of Sound and Vibration. 2014a. Vol. 333, pp. 2421-2442.

[5] M. D. Symans and M. C. Constantinou. Passive fluid viscous damping systems for seismic energy dissipation. ISET Journal of Earthquake Technology, 1998. Vol 35, No.4, pp. 185-206.

[6] S. Raderstrom, M.U-Kaustell, A. Andersson, V. Tell, R. Karoumi. Application of fluid viscous dampers to mitigate vibrations of high-speed railway bridges. International Journal of Rail Transportation. 2016. 